Supercoiled induced transition to the Z-DNA conformation affects the ability of a d(CG/GC) sequence to be organized into nucleosome-cores

\title{
J.M.Casasnovas and F.Azorin*
}

Grupo de Quimica Macromolecular, Centro de Investigacion y Desarrollo del CSIC, ETSIIB, 08028

Barcelona, Spain

Received May 15, 1987; Revised August 25, 1987; Accepted September 25, 1987

\begin{abstract}
Nucleosome-cores were reconstituted by the salt-dialysis method onto closed circular pDHgi6 DNA which contains a $d(C G / G C)_{12}$ sequence. Alternating $d(C G / G C)_{n}$ sequences form left-handed $Z$-DNA readily when contained in negatively supercoiled DNA. We have investigated the ability of the $d(C G / G C)_{12}$ sequence to be organized into nucleosome-cores when stabilized as Z-DNA through negative supercoiling. We have found that nucleosome assembly at the $d(C G / G C)_{12}$ insert is prevented when the sequence is stable in the Z-conformation but it is not affected at all when the sequence adopts the righthanded B-form.
\end{abstract}

\section{INTRODUCTION}

DNA in solution may exist under a variety of different structural conformations (1). DNA sequences containing alternating purine and pyrimidine residues are known to undergo transition to the left-handed $Z$-DNA conformation in response to changing environmental conditions (2). In particular, the repetitive $\mathrm{d}(\mathrm{CG} / \mathrm{GC})_{\mathrm{n}}$ sequence has been shown to form $\mathrm{Z}-\mathrm{DNA}$ when contained in negatively supercoiled DNA $(3,4)$. Other alternating $(5,6)$ and non-strictly alternating (7) DNA sequences can also form Z-DNA through negative supercoiling.

Although no direct evidence for its in vivo existence has yet been obtained, it has been suggested that the left-handed Z-conformation may play an important structural or regulatory function in eukaryotic cells (8). Recent results strongly suggest that Z-DNA is formed transiently during homologous DNA recombination (9). On the other hand, a class of nuclear proteins exists which preferentially recognize the left-handed Z-conformation (10-12). In particular, a protein factor which mediates homologous DNA recombination in Ustilago has been shown to be a Z-DNA 
binding protein (13). Eukaryotic DNA is organized as chromatin. DNA in chromatin is tightly packed into nucleosome-cores which constitute the basic repetitive structural subunit of chromatin organization. Considerations of the possible biological role of Z-DNA in eukaryotes raise the question of the effect of this alternative DNA conformation on the nucleosomal organization of chromatin.

In this paper, nucleosome-cores were reconstituted in vitro by the salt dialysis method onto closed circular pDHgi6 DNA which contains an alternating d(CG/GC) 12 sequence stabilized in the left-handed $\mathrm{Z}$-conformation through negative supercolling. The ability of the alternating $d(C G / G C)_{12}$ sequence to be organized into nucleosomes has been investigated. Others $(14,15)$ have also addressed this question by studying nucleosome reconstitution onto the synthetic chemically modified poly(dG$\mathrm{m}^{5} \mathrm{dC}$ ) copolymer stabilized in the left-handed $\mathrm{z}$-conformation by divalent or multivalent cations. We have found that nucleosome reconstitution at the $d(C G / G C)_{12}$ sequence is prevented when the sequence forms $Z$-DNA but it is not affected at all when the alternating $d(C G / G C)_{12}$ sequence is in the right-handed $B-$ form. The blological significance of these results is discussed. short stretches of alternating $d(C G / G C)_{n}$ sequences are moderately abundant in eukaryotic genomic DNA (16) and they have been shown to enhance homologous recombination of adjacent sequences by as much as 15 fold (17). On the other hand, negative supercoiling is likely to be an important factor determining Z-DNA stability in vivo.

\section{MATERIALS AND METHODS}

\section{Histones and DNA}

Core-histones were obtained from purified chicken erythrocyte nuclei by differential acid extraction according to Johns (18). Purified core-histones were kept at $-20^{\circ} \mathrm{C}$ as an acetone powder. H1/H5 or HMGs proteins were absent and the four corehistones were present to approximately equimolecular amounts.

pDHg16 is a $2.2 \mathrm{~kb}$ plasmid derived from pBR322 which contains a $d(C G / G C)_{12}$ insertion (19). Plasmid DNA was prepared by standard procedures (20). The linking difference ( $T$ ) of 
the purified closed circular DNA was fixed by relaxation with Topoisomerase-I (BRL) in the presence of various concentrations of ethidium bromide (4). Linking differences of the resulting plasmids were determined by the band-counting method (21).

Reconstitution of nucleosome-cores

Nucleosome-core reconstitution was carried out by the salt dialysis method. In summary, purified core-histones were dissolved to a concentration of $2 \mathrm{mg} / \mathrm{ml}$ in $1 \mathrm{M} \mathrm{NaCl}, 5.5 \mathrm{mM}$ EDTA $\mathrm{pH}=7.0$. Histones were then incubated. at room temperature for 30 minutes and mixed with the appropiate amounts of pDHg16 DNA in $2 M$ $\mathrm{NaCl}, 10 \mathrm{mM}$ EDTA $\mathrm{pH}=7.3$. The salt was then removed by stepwise dialysis against solutions of decreasing ionic strength : $1.4 \mathrm{M}$ $\mathrm{NaCl}$ for 3 hrs., 1.2M NaCl for 3 hrs., $1.0 \mathrm{M} \mathrm{NaCl}$ for 3 hrs., $0.85 \mathrm{M} \mathrm{NaCl}$ for $12 \mathrm{hrs}, 0.7 \mathrm{M} \mathrm{NaCl}$ for $3 \mathrm{hrs}, 0.6 \mathrm{M} \mathrm{NaCl}$ for 3 hrs., $0.5 \mathrm{M} \mathrm{NaCl}$ for 3 hrs., $0.4 \mathrm{M} \mathrm{NaCl}$ for 18 hrs. and $0.2 \mathrm{M} \mathrm{NaCl}$ for 6 hrs. The total reconstitution time was approximately 48 hrs. and all dialysis were carried out at $4^{\circ} \mathrm{C}$. Reconstituted complexes were then treated with Topoisomerase-I (BRL) at room temperature for 16-18 hrs. with 15 enzyme units/ng DNA, deproteinized and analyzed by two dimensional gel electrophoresis as described below.

Nuclease digestion experiments

Reconstituted complexes were digested with micrococcal nuclease (SIGMA) at $37^{\circ} \mathrm{C}$ with 0.04 enzyme units(SIGMA)/mg DNA for 30 minutes in a buffer containing $200 \mathrm{mM} \mathrm{NaCl}, 3 \mathrm{mM} \mathrm{CaCl}$, $10 \mathrm{mM}$ Tris-HCl $\mathrm{pH}=7.5$. Digestion was stopped by the addition of EDTA to a final concentration of $15 \mathrm{mM}$. Samples were then deproteinized and the micrococcal nuclease resistant DNA fragments analyzed on a $7 \%$ polyacrilamide-TBE gel. Electrophoresis was carried out at 150 volts for 3 hrs.

Restriction endonuclease digestions were performed at $37^{\circ} \mathrm{C}$ for 45 minutes with 15 enzyme units/ug DNA in a buffer containing $100 \mathrm{mM} \mathrm{NaCl}, 10 \mathrm{mM} \mathrm{MgCl} 2,10 \mathrm{mM}$ Tris-HCl $\mathrm{pH}=7.5$. Digestion was stopped by the addition of EDTA to a final concentration of $15 \mathrm{mM}$. All restriction enzymes were from New England Biolabs.

Two-dimensional gel electrophoresis

Two-dimensional agarose gel electrophoresis was performed 
mainly as described by Peck and wang (22). Gels were formed from a $1 \%$ Agarose-TBE solution and electrophoresed in the first dimension at room temperature in the presence of TBE at 75 volts $(75 \mathrm{~mA})$ for $14 \mathrm{hrs}$. Gels were then equilibrated for $6-8$ hrs. in TBE buffer containing $2 \mu \mathrm{Ng} / \mathrm{ml}$ of chloroquine phosphate (SIGMA) and then electrophoresed in the second dimension at 75 volts (90mA) for 10 hrs. at room temperature in TBE buffer containing $2 \mu \mathrm{g} / \mathrm{ml}$ of chloroquine phosphate. After electrophoresis, chloroquine was removed by soaking the gel in distilled water for 30 minutes. Gels were then stalned with ethidium bromide $(1 \mathrm{Ng} / \mathrm{ml})$ for 30 minutes, destained for 90 minutes in distilled water and photographied under UV light. For quantitation, negatives were scanned in a Joyce-Loebl densitometer. Each spot was scanned in two different directions and its intensity determined from the area contained under the densitometric peak.

\section{RESULTS}

Nucleosome-cores were assembled onto negatively supercolled pDHg16 by the salt reconstitution method as described in Materials and Methods. The fidelity and extent of nucleosome reconstitution were monitored by micrococcal nuclease digestion as well as by measuring the change in linking associated to the formation of nucleosomes.

Efficient nucleosome assembly can be diagnosed by the generation of distinct DNA fragments resistant to nuclease cleavage. The effect of micrococcal nuclease digestion on nucleoprotein complexes obtained at different histone/DNA ratios is shown in Figure 1. A nuclease resistant fragment of about 146 bp. in length is always detected, indicating formation of nucleosomecores. Furthermore, at the highest histone/DNA ratio assayed generation of oligonucleosomal particles is also observed.

Reconstitution of nucleosomes onto a closed circular DNA molecule changes its topological state. Wrapping the DNA around the octameric histone-core modifies both the writhe and the twist of the $146 \mathrm{bp}$. of DNA associated to it. DNA in the core particle is constrained the equivalent of -1 superhelical turn (21,23). In order to compensate for this change in topology of the DNA associated to the nucleosome-core, the molecule should become positi- 


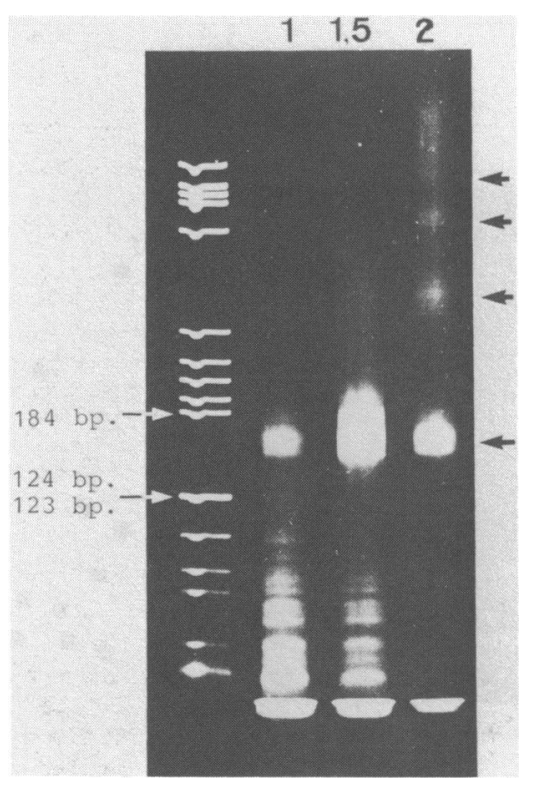

Figure 1.- Micrococcal nuclease digestion of reconstituted complexes obtained at different input histone/DNA ratios, indicated by numbers on top. A 7\% polyacrilamide-TBE gel of the micrococcal nuclease resistant DNA fragments is shown. Lane at the left corresponds to a pBR322 HaeIII digest used as size marker. Black arrows indicate the positions corresponding to core and oligonucleosomal DNA. Fast migrating bands correspond to fragments generated by micrococcal nuclease digestion of naked DNA.

vely supercoiled by as many turns as nucleosomes were introduced in the molecule. Treatment of the resulting nucleoprotein complexes with Topoisomerase-I (Topo-I) will incorporate these topological changes to the DNA itself. Topo-I would restore the reconstituted complexes to the relaxed state by modifying the linking number of the DNA molecule. Upon subsequent deproteinization, supercoils constrained in nucleosomes would be expressed so that deproteinization would yield negatively supercoiled DNA with a linking difference which would reflect exactly the number of nucleosomes originally present in the complex. In other words, if a single nucleosome was introduced in the molecule, the linking difference of the Topo-I treated, deproteinized DNA would be -1 . Complexes differing by one in the number of nucleosomes should produce deproteinized DNA molecules differing also by one in their linking differences. 


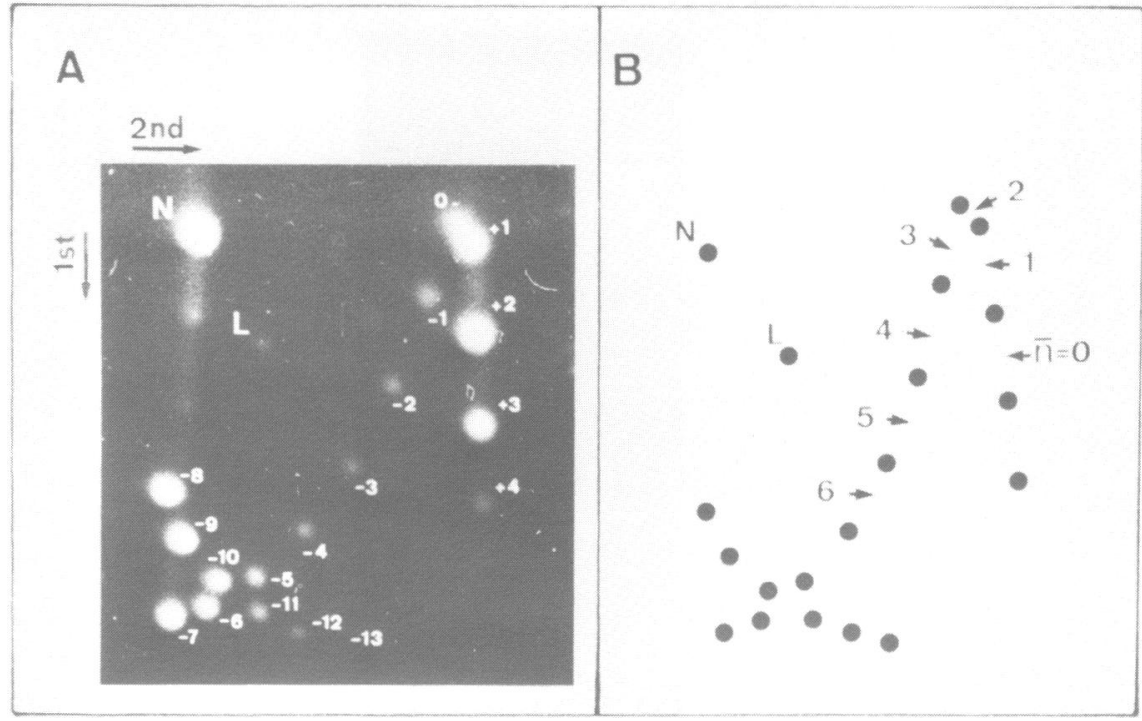

Figure 2.- Nucleosome-core reconstitution onto closed circular pDHg16. (A) Two-Dimensional gel electrophoresis of reconstituted complexes obtained from negatively supercoiled pDHg16 ( $\boldsymbol{\tau}=-12)$ at an input histone/DNA ratio of 1.5 (w/w). After reconstitution, samples were treated with Topo-I, deproteinized and loaded on a two-dimensional $1 \%$ Agarose-TBE gel. First dimension was carried out in TBE buffer and second dimension was run in TBE containing 2Ng/ml of chloroquine phosphate. Numbers indicate the linking difference $(\tau)$ of each topoisomer. (B) Schematic representation of the gel in $A$, showing superimposed a matrix reflecting the population average number of nucleosomes ( $\bar{n})$ present in the reconstituted complexes. $\mathrm{N}=\mathrm{nicked,} \mathrm{L}=\mathrm{linear}$.

Figure 2, shows a typical result obtained from a nucleosome reconstitution experiment. In this case, nucleosomes were assembled into negatively supercolled pDHg16 with an average linking difference (T) of -12 . Nucleosome reconstitution at an input histone/DNA ratio of 1.5 (w/w) followed by Topo-I treatment and deproteinization gives rise to a series of topoisomers which can be resolved by two-dimensional agarose gel electrophoresis. The distribution of topoisomers shown in Figure $2, A$ arises from nucleoprotein complexes which differ in their average number of nucleosomes $(\bar{n})$. The distribution of topoisomers obtained after Topo-I relaxation of protein-free pDHg16, defines the position corresponding to $\bar{n}=0$. Under the electrophoretic conditions shown in Figure 2,A, relaxed pDHgi6 runs as positively supercoiled 
showing a distribution of topoisomers formed by mainly four topoisomers $(\tau=+4,+3,+2$ and +1$)$ with a mean linking difference of $\boldsymbol{T}_{0}=+2.5$. A matrix reflecting the population average number of nucleosomes present in the reconstituted complexes can then be superimposed to the distribution of topoisomers in Figure $2, A$, since each additional nucleosome in the complex reduces the linking difference of the corresponding DNA by one (Figure $2, B$ ). The non-integer value of $\tau_{0}$ reflects the fact that the size of pDHg16 is not a multiple of the helical pitch. As a consequence, the population average number of nucleosomes corresponding to each topoisomer is also fractional, although the actual number of nucleosomes per molecule has to be an integer. For example, topoisomer $\tau=0$ in Figure $2, A$ corresponds to a population average number of nucleosomes $\bar{n}=2.5$. meaning that it is generated by half of the molecules with $n<2$ and half of the molecules with $n>3$. An additional contribution to the heterogeneity of the population of complexes represented by each topoisomer comes from the fact that Topo-I relaxation does not result on a unique topoisomer but instead it produces a Gaussian distribution of topoisomers reflecting the Boltzman's energies distribution function. In our case, relaxation of pDHg16 gives rise to mainly four topoisomers, $\tau=+1,+2,+3$ and +4 in Figure $2, A$, which contribute to the total population by about $10 \%, 40 \%, 40 \%$ and $10 \%$ respectively. Therefore, each topoisomer in Figure $2, A$ would be the result of the contribution due to four different classes of reconstituted complexes differing in their precise number of nucleosomes. For example, topoisomer $\tau=0$ is generated by $10 \%$ of the molecules containing one nucleosome, $40 \%$ containing two nucleosomes, $40 \%$ containing three nucleosomes and $10 \%$ containing four nucleosomes. Figure 2 , also provides information about the $B-Z$ transition of the $d(C G / G C)_{12}$ insert in negatively supercoiled pDHg16. The two-dimensional distribution of topoisomers in Figure $2, A$, shows a sharp break centered around topoisomer $\tau=-8$, which reflects the unwinding due to the formation of $Z-D N A$ within the d(CG/GC) 12 insert. Both the linking difference at which transition occurs as well as the total winding change obtained from the transition are in complete agreement with previously reported results (19). The $\mathrm{d}(\mathrm{CG} / \mathrm{GC})_{12}$ sequence is stable in the $\mathrm{Z}-\mathrm{DNA}$ 


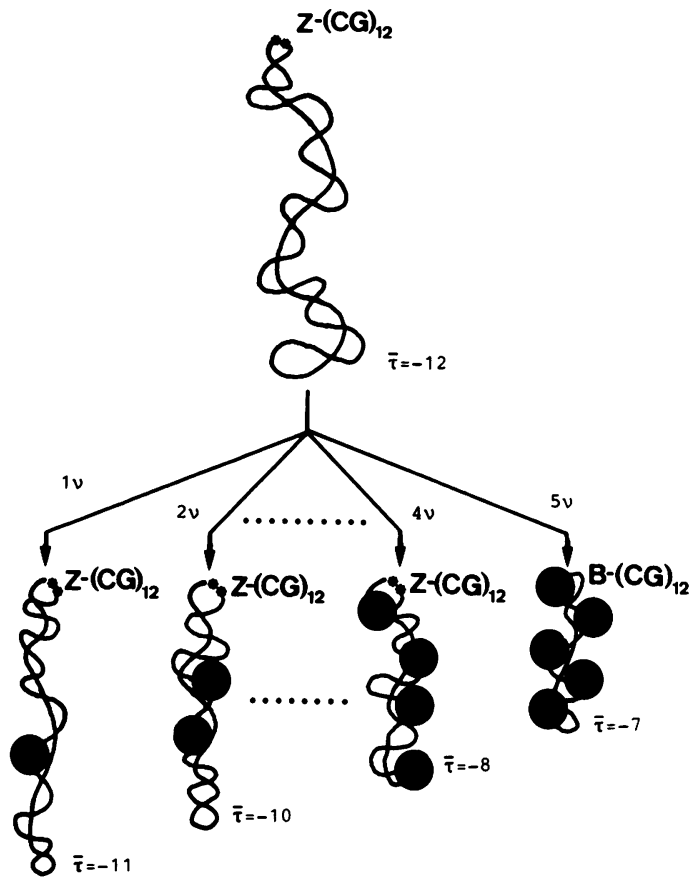

Figure 3.- Effect of nucleosome reconstitution on the stability of the left-handed $Z$-form at the $\alpha(C G / G C)_{12}$ insert in negatively supercoiled pDHg16 $(\bar{\tau}=-12)$. Each nucleosome which gets reconstituted increases by one the effective number of unconstrained supercoils, therefore the $d(C G / G C)_{12}$ would undergo transition to the right-handed B-form after five nucleosomes had been introduced into the DNA molecule. $n=n u c l e o s o m e$, T=linking difference.

conformation whenever $\tau<-8$, but above this value the B-conformation is favoured.

As it is shown in Figure 3 , nucleosome reconstitution onto negatively supercoiled pDHg16 has a dramatic effect on the stability of the Z-conformation at the $d(C G / G C)_{12}$ sequence. In the experiment described in Figure 2 , nucleosomes were assembled onto negatively supercoiled pDHgi6 with $T=-12$. At this superhelicity the $d(C G / G C)_{12}$ insert is stable in the left-handed $z$-conformation (19). However, each nucleosome which gets reconstituted increases by one the effective number of unconstrained supercoils that is, the effective linking difference $\left(\tau_{c}\right)$ of the resulting complex. Therefore, nucleosome reconstitution will 


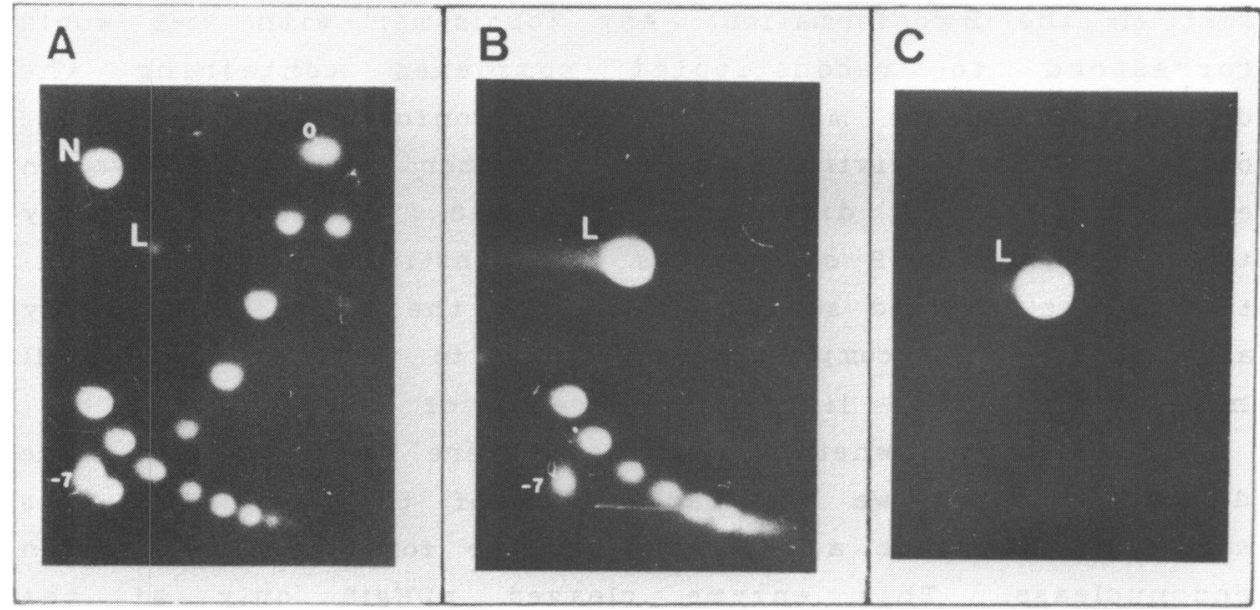

Figure 4.- Effect of the formation of Z-DNA at the d(CG/GC) 12 insert on the cleavage of pDHg16 by several restriction endonucleases. (A) Two-dimensional gel electrophoresis of a mixed topoisomer population obtained by Topo-I relaxation in the presence of different amounts of ethidium bromide. Numbers correspond to the linking difference $(\tau)$ of selected topoisomers. (B) The same population of topoisomers of $A$ but after being digested with BssHII as described in Materials and Methods. (C) Same as $B$ but digestion was carried out with BglI. N=nicked, L=linear.

destabilize the left-handed $\mathrm{Z}$-form at the $d(C G / G C)_{12}$ insert. Transition to the B-conformation will take place in any reconstituted complex contalning five or more nucleosomes, since their effective linking difference $\tau_{c}>-7$ is above the threshold required to stabilize the z-conformation at the $d(C G / G C)_{12}$ insert (19). Therefore, $\bar{n}=5$ in Figure $2, B$ defines the point at which transition to the B-conformation of the $d(C G / G C)_{12}$ sequence should occur in the reconstituted complexes. Taking into account the population origin of each topoisomer in Figure 2 as well as the Gaussian character of the distribution of topoisomers corresponding to the starting negatively supercolled pDHgi6 ( $T=-$ 12), we found that the actual $z$ to $B$ transition of the $d(C G / G C)_{12}$ sequence in the reconstituted complexes should be centered around topoisomer $\tau=-2$. About $50 \%$ of the complexes giving rise to topoisomer $\tau=-2$ would have an effective linking difference of $\tau_{c \geqslant-7}$, thus containing the $d(C G / G C) 12$ in- 
sert in the B-conformation. Any topoisomer with $\tau>-2$ would correspond to reconstituted complexes containing the $d(C G / G C)_{12}$ sequence mainly in the z-conformation (about $80 \%$ of the complexes giving rise to topoisomer $\tau=-1$ would have an effective linking difference of $\left.\tau_{c} \leqslant-8\right)$. Concurrently, any topoisomer with $\tau<-2$ corresponds to reconstituted complexes containing the inserted sequence mainly in the B-conformation conly about $20 \%$ of the complexes giving rise to topoisomer $T=-3$ still have an effective linking difference of $\left.\tau_{c}<-8\right)$.

To determine whether nucleosomes were reconstituted at the d(CG/GC) 12 insert we took advantage of the fact that this sequence constitutes a recognizition site for BssHII restriction endonuclease. This enzyme cleaves pDHg16 only at the $d(C G / G C)_{12}$ insert and it is sensitive to the conformational state of the recognizition site (24). Cleavage of pDHg16 with BssHII is strongly inhibited by the formation of Z-DNA within the d(CG/GC $)_{12}$ sequence. As it is shown in Figure 4,B, any toporsomer with $\tau>-7$, thus containing the $\mathrm{d}(\mathrm{CG} / \mathrm{GC})_{12}$ insert in the B-conformation, is completely linearized by BssHII after a 45 minutes incubation period. On the other hand, topoisomers with $\tau<-7$ which contain the $d(C G / G C)_{12}$ sequence in the $Z$-conformation, are resistant to BssHII cleavage. It is interesting to note that topoisomer $\tau=-7$ is partially resistant to BssHII cleavage although, under these electrophoretic conditions, the $d(C G / G C)_{12}$ insert is not stable in the z-conformation. Ionic conditions and, in particular divalent cations are known to affect strongly the B-Z equilibrium $(25,26)$. It is possible that under the ionic conditions at which BssHII digestion is carried out, namely $100 \mathrm{mM} \mathrm{NaCl}, 10 \mathrm{mM} \mathrm{MgCl}_{2}$, the $\mathrm{d}(\mathrm{CG} / \mathrm{GC})_{12}$ insert may be already stable in the z-conformation at this linking difference and therefore resistant to BssHI cleavage. Alternatively, it is also possible that at this superhelicity the d(CG/GC) 12 sequence would be flipping to the z-conformation back and forth, interfering with BssHI cleavage.

Digestion by BglI restriction endonuclease which cleaves PDHg16 outside the $d(C G / G C)_{12}$ insert is not affected by the formation of Z-DNA within this sequence (Figure 4,C).

Reconstitution of nucleosomes at the $\mathrm{d}(\mathrm{CG} / \mathrm{GC})_{12}$ insert 


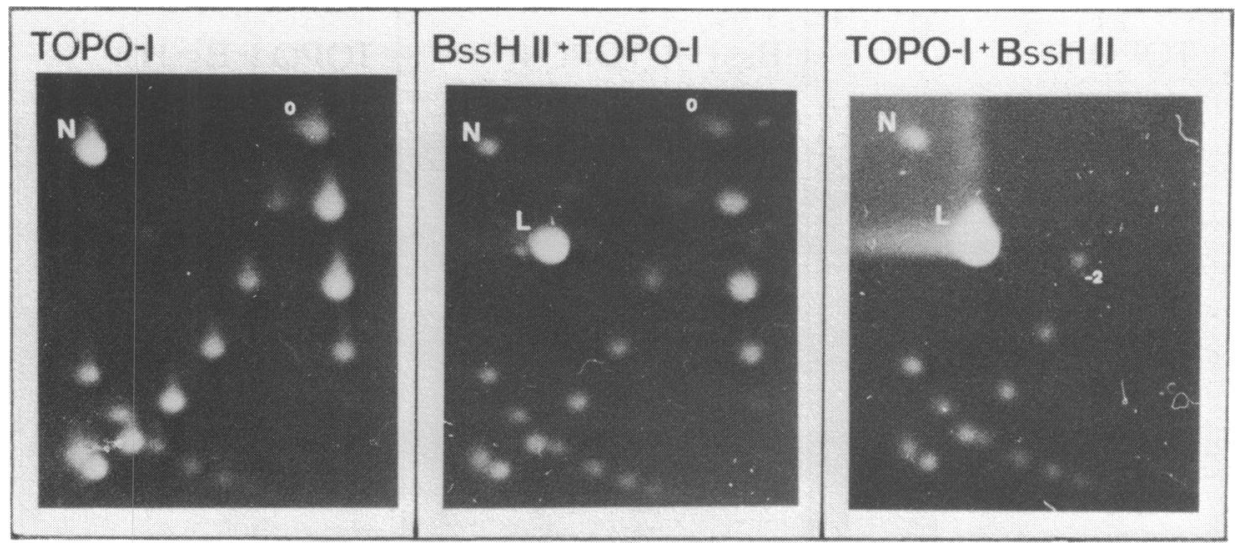

Figure 5.- Cleavage by BssHII of reconstituted complexes obtained from negatively supercoiled pDHg16 ( $F=-12)$. Complexes were digested with BssHII either before (middle panel) or after Topo-I treatment (right panel). Left panel shows the two-dimensional distribution of topoisomers obtained from undigested reconstituted complexes. Numbers correspond to the linking difference ( $\tau$ )

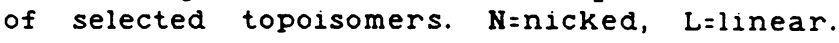

should result in a decreased accessibility of the reconstituted complexes to BsshII cleavage. The more nucleosomes present in the complexes the more protected from BssHII cleavage the sequence would be. Figure 5, shows the accessibility to BssHII of reconstituted complexes obtained from negatively supercoiled pDHg16 $(\bar{\tau}=-12)$ as described in Figure 2 . As shown in Figure 5 , right panel, reconstituted complexes containing less than five nucleosomes in average, become freely accessible to BssHII when they are first treated with Topo-I to release any unconstrained supercoils, and then subjected to BssHII action. On the other hand, the same complexes show a marked resistance to cleavage when they are subjected to BssHII cleavage prior to Topo-I relaxation (Figure 5, middle panel). Even topoisomers arising from complexes containing none or very few nucleosomes are markediy resistant to BssHII cleavage, indicating that the $d(C G / G C)_{12}$ insert in these complexes is stable in the Z-conformation prior to Topo-I relaxation and thus, they are resistant to BSSHII cleavage regardless of nucleosome protection. After Topo-I treatment, all reconstituted complexes would contain the $\mathrm{d}(\mathrm{CG} / \mathrm{GC})_{12}$ insert in the B-conformation. Therefore, BSSHII 


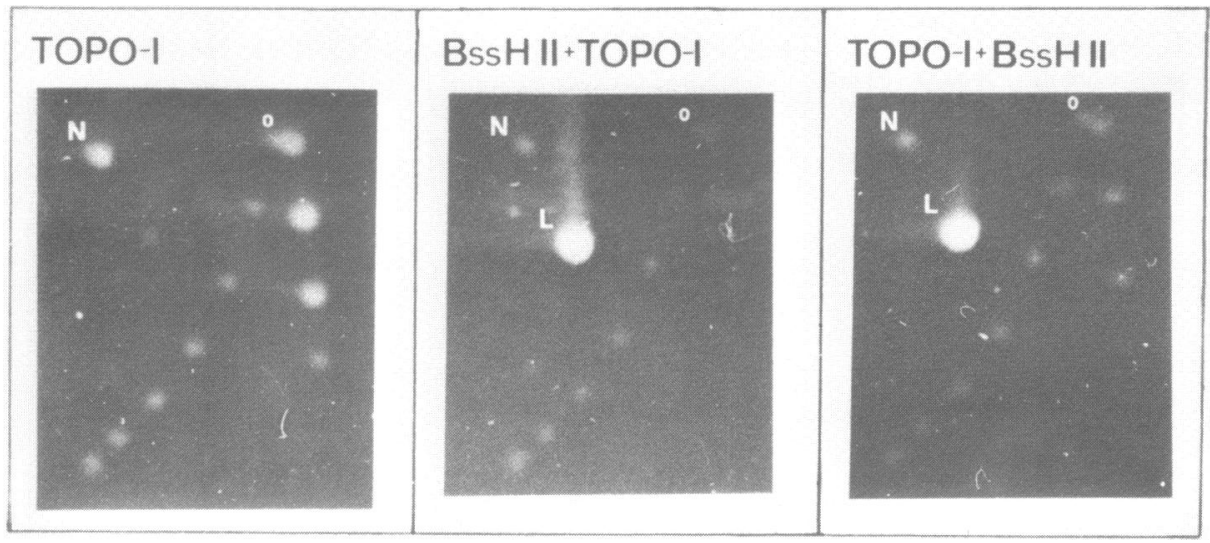

Figure 6.- Cleavage by BssHII of reconstituted complexes obtained from relaxed pDHg16. Complexes were digested with BssHII elther before (middle panel) or after (right panel) Topo-I relaxation. Left panel shows the distribution of topoisomers obtained from undigested complexes. Numbers correspond to the linking

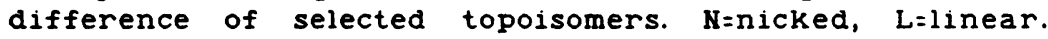

cleavage in this case is not affected by the conformational state of the sequence and reflects precisely the degree of protection due to nucleosome deposition at the $d(C G / G C)_{12}$ sequence. Interestingly, the electrophoretic pattern obtained in this case (Figure 5, right panel), shows a sharp transition from no-protection to protection occurring at topoisomer $\tau=-2$ which, as we have mentioned earlier, coincides with the point at which transition to the B-conformation of the $a(C G / G C)_{12}$ insert in the reconstituted complexes is expected. The relative degree of protection from BssHI cleavage after Topo-I treatment for each topoisomer in Figure 5, right panel, can be determined from the ratio of its intensity after Topo-I treatment and BssHII digestion (ITopo+BssH) divided by its intensity after Topo-I treatment alone (ITopo). As it is shown in Figure $7, A$, any topoisomer with $T>-2$ is highly sensitive to BsshII digestion, showing that complexes which prior to Topo-I treatment contained the $d(C G / G C)_{12}$ sequence stable in the z-conformation, as shown by the results in Figure 5, middle panel, are not protected from endonucleolitic cleavage. This result strongly suggests that when the $\alpha(C G / G C)_{12}$ sequence is stable in the left-handed $z$ conformation, nucleosomes are extruded from the sequence so that 

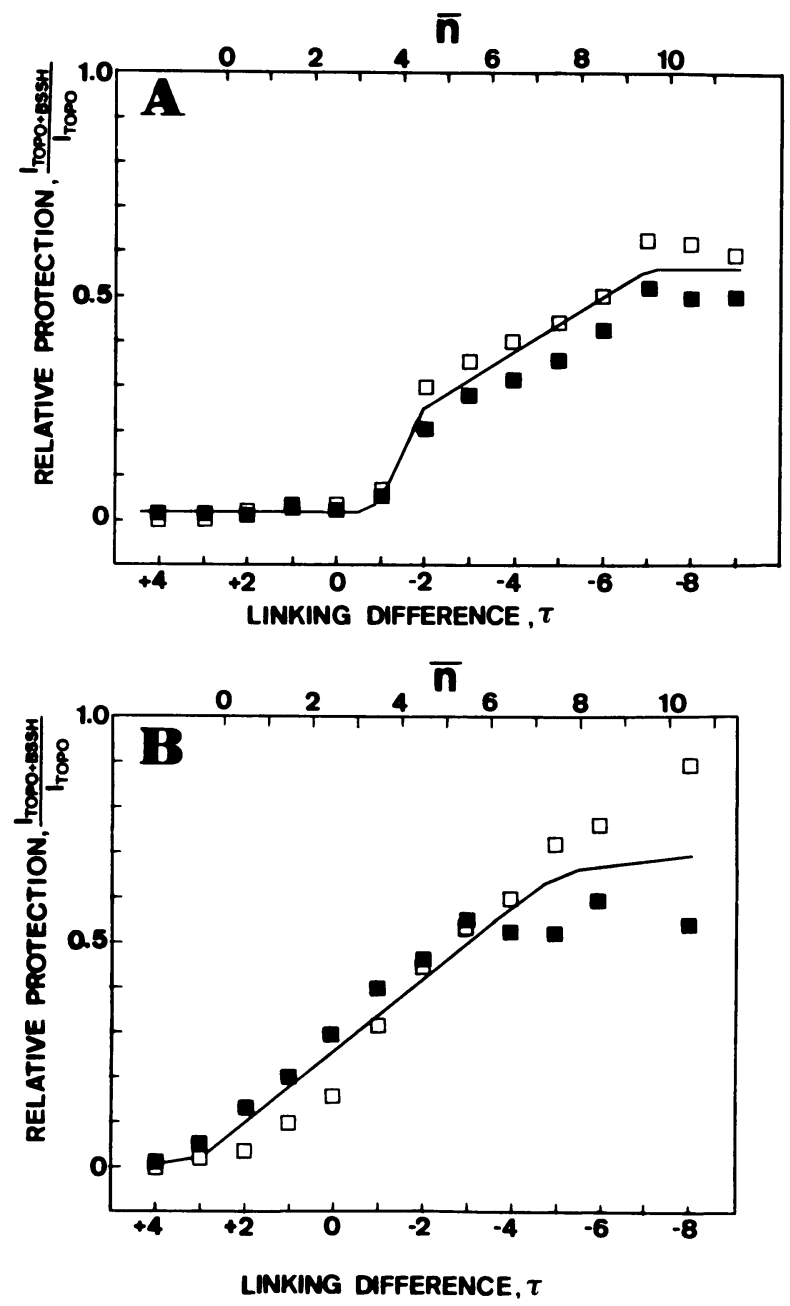

Figure 7.- The relative protection from BssHII cleavage after Topo-I treatment is shown as a function of the average number of nucleosomes $(\bar{n})$ for reconstituted complexes obtained from negatively supercoiled pDHg16 $(T=-12)(A)$ and relaxed pDHg16 (B). The relative degree of protection for each topoisomer was determined from the ratio of its intensity after sequential treatment with Topo-I and BssHII (I Topo+BssH) divided by its intensity after Topo-I treatment alone (ITopo). The results of two independent experiments (indicated by solid and open symbols) are presented. $\tau$ is the linking difference.

complexes become freely accessible to BssHI after the $\mathrm{d}(\mathrm{CG} / \mathrm{GC})_{12}$ sequence is converted to the B-conformation by Topo-I treatment. When five or more nucleosomes had been intro- 
duced into the DNA molecule so that the $d(C G / G C)_{12}$ sequence is no longer stable in the $\mathrm{z}$-conformation in the reconstituted complexes, nucleosomes are formed at the sequence which now becomes protected from nuclease degradation after Topo-I treatment as indicated by the marked resistance to BssHII cleavage shown by topoisomers with $T<-2$ (Figure $7, A$ ). The degree of protection from BSSHII cleavage levels off after about 9 nuclecsomes have been reconstituted, indicating that when $\bar{n}>8$ inhibition of BssHI cleavage is maximum and independent of the actual number of nucleosomes. Consistently with our interpretation, the precise topoisomer at which BsSHI protection begins, depends on the actual linking difference of the starting negatively supercoiled PDHgi6 used for the reconstitution experiment. When nucleosomes were assembled onto negatively supercoiled PDHgit with a $i=-20$, significant protection was observed after 7-8 nucleosomes were reconstituted (data not shown) which is very close to the average number of nucleosomes at which maximum protection is observed (Figure 7).

When nucleosomes are assembled onto relaxed pDHg16, which contains the $d(C G / G C)_{12}$ sequence in the B-conformation (19), a completely different pattern of Bssil cleavage is obtained (Figure 6). When Bssil digestion is performed after Topo-I treatment (Figure 6, right panel), no sharp transition like that shown for negatively supercoiled pDHgi6 (Figure 5, right panel), is observed. Instead, a progressive protection to BssHII cleavage is detected as the number of nucleosomes present in the reconst1tuted complexes increases (Figure 7,B). The slope of the curve in Figure $7, B$ is very similar to the slope of the upper part of the curve shown in Figure 7,A which also reflects a progressive protection. Assuming a random sequence-independent reconstitution of nucleosomes, any 24 bp. long sequence in pDHg16 should be protected from nuclease attack by about $10 \%$ per each nucleosome present in the reconstituted complex. Within the experimental error, the results shown in Figure $7, B$, are in good agreement with this estimate. Generation of positive supercoiling might account for the small differences detected when BssHI cleavage is performed before Topo-I treatment (Figure 6, middle panel). From these results we conclude that nucleosome reconstitution at 


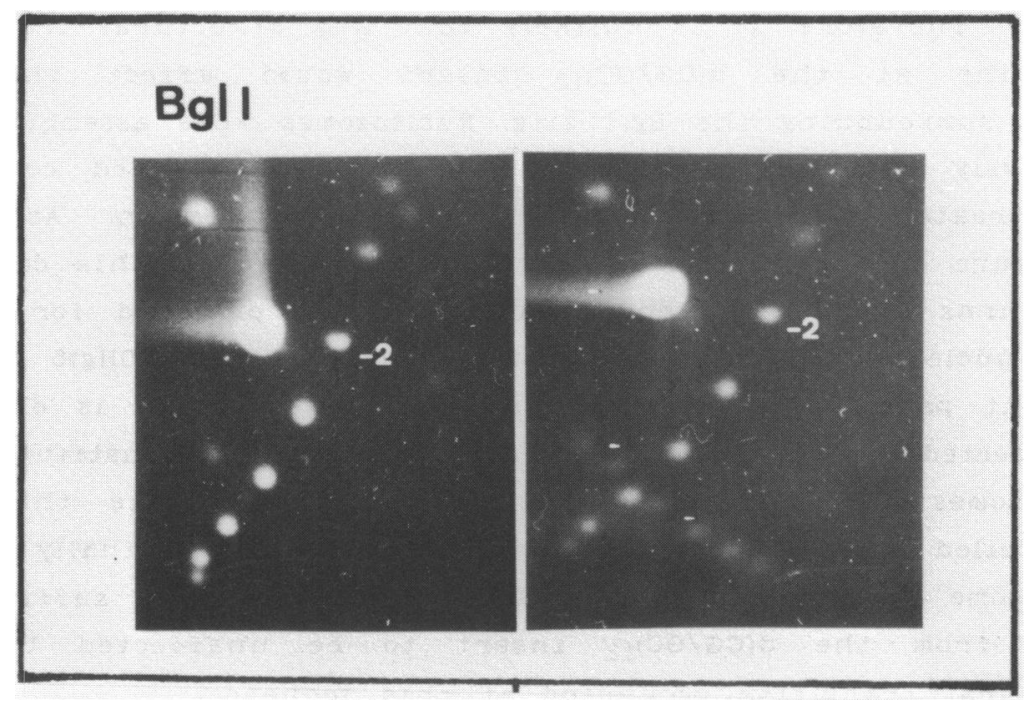

Figure 8.- Cleavage by BglI of reconstituted complexes obtained from negatively supercolled pDHg16 $(T=-12)$. Complexes were treated with Topo-I prior to nuclease digestion. Numbers correspond to the linking difference of selected topoisomers. Results obtained from two independent experiments are shown.

the $d(C G / G C)_{12}$ sequence is not affected at all by the repetitive nature of the sequence as long as it is maintained in the $B$ conformation.

After a similar number of nucleosomes have been reconstituted, complexes obtained from relaxed pDHg16 show a slightly higher degree of protection than complexes obtained from negatively supercoiled pDHg16 (Figure 7). Several interpretations for this result are possible. Small systematic differences in the amount of DNA loaded in the gels might account for these variations. It is also possible that this differential protection might arise from a preferential deposition of nucleosomes at the region flanking the $d(C G / G C)_{12}$ insert while the sequence is stable in the Z-conformation.

The pattern of protection from cleavage by nucleases described in Figure 5, right panel for BssHII is characteristic of the $d(C G / G C)_{12}$ insertion in pDHg16. Figure 8 , shows the pattern of digestion obtained for BglI restriction endonuclease which cleaves pDHg16 about 910 bp. away from the $d(C G / G C)_{12}$ 
insert. Therefore, it is unlikely that any structural transition occurring at the d(CG/GC) 12 insert would affect the DNA region surrounding the BglI site. Nucleosomes were assembled onto negatively supercoiled pDHg16 $(\tau=-12)$ and reconstituted complexes were treated with Topo-I prior to nuclease digestion. As shown in Figure 8, the pattern of digestion obtained in this case for BglI turns out to be very similar to that obtained for BssHII when nucleosomes were reconstituted into relaxed pDHg16 (Figure 6, right panel). A pattern of progressive protection is observed as expected for a random sequence-independent reconstitution of nucleosomes in this region. These results indicate that the supercolled state of the DNA by itself does not actually affect nucleosome reconstitution in a DNA region which is sufficiently apart from the $d(C G / G C)_{12}$ insert to be unaffected by any structural transition occurring at this sequence.

\section{DISCUSSION}

In this paper, we have investigated the ability of a $d(C G / G C)_{12}$ sequence to be organized into nucleosome-cores when stabilized in the left-handed $\mathrm{Z}$-conformation through negative supercoiling. Alternating $d(C G / G C)_{n}$ sequences can form Z-DNA readily when contained in negatively supercoiled DNA $(3,4,19)$. We have found that nucleosome assembly at this simple repeating DNA sequence is prevented as long as the sequence remains in the $\mathrm{z}$-conformation. This conclusion is based on our observation that, no matter how many nucleosomes were reconstituted into the DNA, nucleoprotein complexes containing the $d(C G / G C)_{12}$ sequence in the z-conformation become freely accessible to cleavage by BssHI at the d(CG/GC) 12 sequence after Topo-I treatment. Therefore, when reconstituted complexes are subjected to BssHI digestion after Topo-I treatment, a characteristic sharp transition from no-protection to protection is found associated to the $Z$ to $B$ transition of the $d(C G / G C)_{12}$ insert in the reconstituted complexes (Figure 5, right panel and Figure 7,A). This peculiar pattern of cleavage is not found when nucleosomes are reconstituted onto relaxed pDHg16 which contains the $d(C G / G C)_{12}$ insert in the B-conformation. In this case, a progressive protection to BssHI cleavage is 
obtained as the number of nucleosomes increases (Figure 6, right panel and Figure 7,B). Likewise, a pattern of progressive protection is also obtained when nucleosome reconstitution at a DNA region far apart from the $d(C G / G C)_{12}$ insert is investigated (Figure 8). These results indicate that neither the supercoiled state of the DNA nor the repetitive character of the d(CG/GC) 12 insert are responsible for the peculiar BssHII cleavage pattern shown in Figure 5, right panel.

others have also studied the in vitro reconstitution of nucleosomes onto Z-DNA (14,15). They have investigated the ability to assemble nucleosome-cores onto the linear alternating purine/pyrimidine poly(dG-m5dc) copolymer stabilized in the left-handed $\mathrm{Z}$-form by divalent or multivalent cations. Because of the ionic strength dependency of the $B-Z$ transition in poly(dG$\left.\mathrm{m}^{5} \mathrm{dc}\right)$ (26), nucleosome reconstitution onto this polymer has to be carried out under rather stringent ionic conditions, namely at very low NaCl concentration in the presence of either micromolar concentrations of hexamincobalt or milimolar concentrations of magnesium. In this paper, we have extended these studies to a rather more biologically significant situation. We have studied nucleosome formation onto a $d(C G / G C)_{12}$ sequence when it is stabilized in the z-conformation through negative supercolling, a factor which is believe to play an important role on the in vivo stablization of Z-DNA (2). Furthermore, short stretches of $d(C G / G C)_{n}$ sequences are present to a moderate extent in eukaryotic genomes (16) and they have been shown to enhance homologous recombination (17). Finally, instead of confronting histone-cores with DNA molecules which are fully in the Z-form, we have studied nucleosome reconstitution onto a DNA molecule which contains both Z-DNA and B-DNA, as it is more likely to reflect the in vivo situation.

our results are mainly in agreement with those obtained by Nickol et al. (14), since we were unable to detect nucleosome formation at the $d(C G / G C)_{12}$ insert when adopting the lefthanded z-conformation. We cannot exclude the possibility that nucleosome assembly at the $z$-sequence had occurred but to a low extent going undetected by our techniques. However, from our results we estimate that the relative affinity of the octameric 
histone-core for B-DNA is at least 50 to 100 fold greater than for Z-DNA.

Miller et al. (17), had claimed that nucleosomes can be assembled successfully into Z-DNA though to a low extent when reconstitution is carried out in the presence of nucleoplasmin. However, nucleoplasmin or anyother polyanion, appears to be required when nucleosome assembly is carried out at low lonic strength or under conditions which favour aggregation. Wher salt reconstitution methods are used no such requirement is found and successful nucleosome assembly has been obtained in the absence of nucleoplasmin for a variety of synthetic (27,28) and natural polydeoxinucleotides, including both linear (29-32) and closed circular molecules $(23,33,34)$, the supercolled state of which does not influence the fldelity of nucleosome reconstitution (34).

Z-DNA has a markedly different charge distribution than $B-$ DNA (8). Transition to the left-handed Z-conformation is likely to have a strong effect on the precise DNA-protein electrostatic interactions which govern the stability of the nucleosome-core particle. The inability of Z-DNA to be organized into nucleosomes might also be related to its stiffness. DNA in the core particle is bent to a significant extent (35). Light-scattering studies on the Z-form of poly(dG-dC) have shown that Z-DNA is stiffer and has a greater persistance length than B-DNA (30). Trerefore it is more difficult to bent than B-DNA.

Recognizition of functional DNA sequences in chromatin may be facilitated by nucleosome destabilization. Disruption of the regular nucleosomal organization of chromatin, as judged by the appearance of characteristic nuclease sensitivity fatterns, appears to be a neccesary event for a variety of biological processes incluaing gene activation. Potentially z-forming sequences are found widely spread in eukaryotic DNA (see 2 for a review). In particular, several promoter regions have been shown to contain sequences which undergo transition to the Z-conformation on negatively supercolled DNA (2). Moreover, the repetitıve potentially z-forming sequences $d(C A / G T)_{n}$ and $d(C G / G C)_{n}$ have been shown to be hot-spots for recombination (17,37). Eventual formation of Z-DNA would disrupt nucleosomal organization at 
these sequences facilitating its interaction with trans-acting regulatory proteins which might also recognize specifically tre left-handed z-conformation.

\section{ACKNOWLEDGEMENTS}

We are thankful to Dr. D.E.Pulleyblank for plasmids and to Drs. J.A.Subirana and L.Cornudella for revising the manuscript. The technical assistance of the Taller de la catedra de Tecnologia Mecanica is acknowledged. This work was supported by grants from the CAYCIT, the CSIC and the CIRIT. JMC was a recipient of a doctoral fellowship from the Ministerio de Educacion $y$ Ciencia.

*To whom correspondence should be addressed

\section{REFERENCES}

(1) Zimmerman, S. B. (1982) Ann. Rev. Biochem. 51, 395-427.

(2) Rich, A., Nordheim, A. and Wang. A. H. -J. (1984) Ann. Rev. Biochem. $53,791-846$.

(3) Klysik, J. , Stirdivant, S. M. , Larson, J. E. , Hart, P. A. and Wells, R. D. (1981) Nature 290, 672-677.

(4) Peck, L. J., Nordhe im, A., Rich, A. and Wang, J. (1982) Proc. Natl. Acad. Sci. USA $79,4560-4564$.

(5) Hanlford, D. B. and Pulleyblank, D. E. (1983) Nature 302, 632-634.

(6) Nordheim, A. and Rich, A. (1983) Proc. Nat1. Acad. Sci. USA 80 , $1821-1825$.

(7) Ellison, M. J. , Kelleher III, R. J., Wang. A. H. - J. , Habener, J. F. and Rich, A. (1985) Proc. Nat1. Acad. Sci. USA 82, $8320-8324$.

(8) Wang, A. H. -J. , Quigley, G. J. , Kol pack, F. J. , Crawford, J. L. , van Boom, J. H. , van der Mare 1, G. and Rich, A. (1979) Nature 282, $680-686$.

(9) Kmiec, E. and Holloman, W. K. (1984) Cell 36,593-598.

(10) Nordhe $1 \mathrm{~m}$, A. , Tesser, P., Azorin, F. , Kwon, Y. H. , Moller, A., and Rich, A. (1982) Proc. Nati. Acad. Sc1. USA 79, 7729-7733.

(11)Azorin, F. and Rich, A. (1985) Cell 41, 365-374.

(12) Lafer, E. M. , Sousa, R., Rosen, B. , Hsu, A. and Rich, A. (1985) Biochemistry 24,5070-5076.

(13) Kmiec, E., Angelides, K. J. and Holloman, W. K. (1985) Cell 40, $139-145$.

(14) Nickol, J. , Behe, M. and Felsenfeld, G. (1982) Proc. Natl. Acad. Sci. USA 79, 1771-1775.

(15)Miller, F. D. , Dixon, G. H., Rattner, J. B. and van de Sande, J. (1985) Biochemistry 24,102-109.

(16) Hamada, H. , Petrino, M. G. and Kakunaga, T. (1982) Proc. Nat1. Acad. Sc1. USA 79, 6465-6469.

(17) Bullock, P., Miller, J. and Botchan, M. (1986) Mol.Cell Biol. 6, $3954-3964$.

(18) Johns, E. W. (1964) Biochem. J. 92, 55-59.

(19) Haniford, D. B. and Pulleyblank, D. E. (1983) J. Blomol. Str. Dynam. $1,593-609$. 
(20) Maniatis, T. Fritsch, E. F. and Sambrook, J. (1982) Molecular Cloning. (Cold Spring Harbor, New York). Cold Spring Harbor Laboratory.

(21) Keller, w. (1975) Proc. Natl. Acad. Sci. USA 72, 4876-4880.

(22) Peck, L. J. and Wang, J. (1983) Proc. Nat1. Acad. Sci. USA 80, 62066210.

(23) Simpson, R. T., Thoma, F. and Brubaker, J.M. (1985) Ce11 42,799808.

(24) Azorin, F., Hahn, R. and R1ch, A. (1984) Froc. Nat?. Acad. Sc1. USA $81,5714-5718$.

(25) Azorin, F., Nordheim, A. and Rich, A. (1983) EMBO J. 2,649-655.

(26) Behe, M. and Felsenfeld, G. (1981) Proc. Natl. Acad. Scl. USA 78 , $1619-1623$.

(27) Simpson, R. T. and Kunzlar, P. (1979) Nucleic Acids Res. 6,13871415.

(28) Bryan, P. N. , Wright, E. B. and Olins, D. E. (1979) Nucleic Acids Res. 8,2751-2769.

(29) Axel, R. , Melchior, W. , Sollner-Webb, B. and Felsenfeld, G. (1974) Proc. Nat1. Acad. Sci. USA 71, 4101-4105.

(30) Kornberg, R. D. and Thomas, J. O. (1974) Science 184,865-868.

(31) Boseley, G. , Bradbury, M. F. , Butler-Brown, G. S. , Carpenter, B. G. and Stephens, R. M. (1976) Eur. J. Biochem. 62, 21-31.

(32) Woodcock, C. F. L. (1977) Science 195, 1350-1352.

(33) Oudet, P., Gross-Bellard, M. and Chambom, P. (1975) Cell 4,281300.

(34) Germond, J. E. , Hirt, B. , Oudet, P. , Gross-Bellard, M. and Chambon, P. (1975) Proc. Nat1. Acad. Sci. USA 72, 1843-1847.

(35) Richmond, T. J., Finch, J. T., Rushton, B., Rhodes, D. and Klug, A. (1984) Nature 311,532-537.

(36) Thomas, J. T. and Bloomfield, V. A. (1983) Nucleic Acids Res. 11, $1919-1930$.

(37) Treco, D. and Arnheim, N. (1986) Mol. Cell B101. 6, 3934-3937. 